\title{
Mediastinal Germ Cell Tumors in Pediatric Patients: A Report From the Italian Association of Pediatric Hematology and Oncology
}

\author{
Maria Debora De Pasquale, $\mathrm{MD}, \mathrm{PhD}_{,}{ }^{1}$ Alessandro Crocoli, $\mathrm{MD},{ }^{2,3 *}$ Massimo Conte, $\mathrm{MD}^{4}$ Paolo Indolfi, $\mathrm{MD},{ }^{5}$ \\ Paolo D'Angelo, $\mathrm{MD}^{6}{ }^{6}$ Renata Boldrini, $\mathrm{MD},{ }^{7}$ Monica Terenziani, $\mathrm{MD}^{8}{ }^{8}$ and Alessandro Inserra, $\mathrm{MD}^{9}$
}

Background. Primary mediastinal germ cell tumors (GCTs) are rare in children and still represent a challenge for both adult and pediatric oncologists because of their worse outcome compared to their gonadal counterpart. Procedure. Prospectively collected data concerning patients enrolled in the Italian Association of Pediatric Haematology and Oncology study on malignant GCTs (AIEOP TCGM 2004) protocol for the treatment of GCTs were analyzed. Patients with malignant mediastinal primary GCTs were included in this study. Data regarding patients with newly diagnosed mediastinal teratoma were also collected. Results. From 2005 to 2013, 20 children diagnosed with mediastinal GCTs were registered in
AIEOP TCGM 2004 protocol. With a median follow-up of 89 months (range 35-123), the overall survival (OS) and event free survival (EFS) rates were $100 \%$ for teratoma and $90 \%$ for malignant GCTs. Conclusions. We confirm the favorable outcome of children affected by mediastinal teratoma and malignant GCTs. For malignant tumors, further studies on the clinical characteristics and genetic signatures on tumor samples might be necessary to better understand differences observed in high-risk patients and to assist the development of more effective treatment for this subgroup. Pediatr Blood Cancer 2016;63:808-812. (c) 2016 Wiley Periodicals, Inc.

Key words: childhood; germ cell tumors; malignancies; mediastinal tumors; teratoma

\section{INTRODUCTION}

Primary mediastinal germ cell tumors (GCTs) are rare in children, accounting for only $6-18 \%$ of pediatric mediastinal neoplasms. In this respect, published studies regarding these tumors include either pediatric cases extracted from adult series or children cared for over a long period with treatment modalities varying over the years. This confirms the rarity of the disease and explains the low number of patients available for analysis.[1-3]

Even though treatment of pediatric GCTs is well established, mediastinal GCTs still represent a challenge for both adult and pediatric oncologists. Unlike their gonadal counterpart, mediastinal CGTs often require major surgical procedures to achieve adequate resection: infiltration of vital organs and vessels is a nonnegligible risk factor to be considered prior to surgery, even if resection is in most cases a critical element for favorable outcome.[4] Nevertheless, data on surgical outcome in treatment of both teratoma and malignant pediatric mediastinal GCTs are still lacking, especially regarding patients enrolled in a specific protocol and subsequently diagnosed and treated over a short period of time.

After the first Italian study,[5] we developed guidelines for the treatment of teratoma in conjunction with the second national prospective Italian Association of Pediatric Haematology and Oncology (AIEOP) study on malignant GCTs, referred to as TCGM 2004.

We report here a series of 20 consecutive children diagnosed with mediastinal malignant GCTs and teratoma treated according to AIEOP TCGM 2004 and guidelines for the treatment of teratoma.

\section{MATERIALS AND METHODS}

Prospectively collected data regarding patients enrolled in the AIEOP TCGM 2004 protocol for treatment of GCTs were analyzed. Patients with malignant mediastinal primary GCTs were included for analysis in this study. According to the World Health Organization/International Agency for Research on Cancer classification, malignant GCTs include seminoma, embryonal carcinoma, yolk sac tumor (YST), choriocarcinoma, and teratoma with somatic-type malignancies. [6]

Data on patients with newly diagnosed mediastinal teratoma were collected. Data regarding the histopathological subtype of primary tumor, treatment applied, final status, and followup were collected and analyzed. The staging system used in this protocol for malignant tumors considers stage I as a tumor that has been completely excised, stage II as a tumor completely excised but with microscopic residual, stage III as biopsy only or gross residual tumor, and stage IV as metastatic disease. All initial tumor specimens were evaluated by central pathologic review. Serum levels for alpha-fetoprotein $(\alpha \mathrm{FP})$ and $\beta$ human chorionic gonadotropin $(\beta \mathrm{HCG})$ were also evaluated prior to biopsy/radical surgery. Preoperative imaging (computed

$\overline{{ }^{1} \text { Department }}$ of Oncohematology, Bambino Gesù Pediatric Hospital IRCCS, Rome, Italy; ${ }^{2}$ Operative Unit of General and Thoracic Surgery-Surgery Regional Network, Bambino Gesù Pediatric Hospital IRCCS, Rome, Italy; ${ }^{3}$ Azienda Regionale Ospedaliera San Carlo, Via Potito Petrone snc, Potenza, Italy; ${ }^{4}$ Department of Hematology-Oncology, Giannina Gaslini Children's Hospital, Genoa, Italy; ${ }^{5}$ Pediatric Oncology Unit, Department of Pediatrics, Second University of Naples, Naples, Italy; ${ }^{6}$ Pediatric Hematology and Oncology Unit, G. Di Cristina Children's Hospital, A.R.N.A.S. Ospedale Civico, Palermo, Italy; ${ }^{7}$ Department of Pathology, Bambino Gesù Pediatric Hospital IRCCS, Rome, Italy; ${ }^{8}$ Pediatric Oncology Unit, Fondazione IRCCS Istituto Nazionale dei Tumori, Milano, Italy; ${ }^{9}$ Operative Unit of General and Thoracic Surgery, Bambino Gesù Pediatric Hospital IRCCS, Rome, Italy

Conflict of interest: Nothing to declare.

Maria Debora De Pasquale and Alessandro Crocoli contributed equally to this work.

*Correspondence to: Alessandro Crocoli, Operative Unit of General and Thoracic Surgery-Surgery Regional Network, Bambino Gesù Pediatric Hospital IRCCS, Piazza S. Onofrio 4, 00165 Rome, Italy.

E-mail: alessandro.crocoli@opbg.net

Received 23 July 2015; Accepted 11 December 2015 
TABLE I. Population of Mediastinal Teratoma

\begin{tabular}{lcclc}
\hline Patient & Sex & $\begin{array}{c}\text { Age at } \\
\text { diagnosis } \\
\text { (years, months) }\end{array}$ & \multicolumn{1}{c}{ Symptoms } & $\begin{array}{c}\text { Immature } \\
\text { teratoma }\end{array}$ \\
\hline 1 & M & 2,0 & Abdominal pain & No \\
2 & M & 9,7 & Abdominal pain, fever & Grade 2 \\
3 & M & 9,8 & Fever, cough & No \\
4 & F & 0,5 & Fever, cough & No \\
5 & F & 3,5 & No & No \\
6 & M & 0,1 & Respiratory distress & Grade 2 \\
7 & M & 0,6 & Recurrent pneumonia & Grade 1 \\
8 & M & 5,0 & No & No \\
9 & F & 16,0 & No & No \\
10 & F & 0,1 & Respiratory distress & No \\
\hline
\end{tabular}

tomography (CT) and magnetic resonance imaging (MRI)) was used to evaluate the feasibility of primary tumor resection. All patients with unresectable tumor underwent an initial tissue diagnosis on specimens obtained by biopsy (image-guided procedure or minimally invasive surgical biopsy). In the case of contraindications for general anesthesia, only serum tumor markers were considered for the diagnosis. Attention was focused on the following items: surgical approach, localization, major vessel involvement (aorta, superior vena cava, brachiocephalic artery/vein, pulmonary artery/vein), intraoperative complications, postoperative complications, and outcome.

\section{RESULTS}

From 2005 to 2013, 370 children with GCTs of all sites were registered in AIEOP TCGM 2004 protocol. Twenty patients (13 males and seven females) were diagnosed with mediastinal GCTs. Median age at diagnosis was 3 years and 6 months (range: 8 months to 17 years and 8 months). Ten patients were diagnosed with a malignant tumor and 10 with teratoma. Eighteen of the 20 patients presented with initial symptoms of respiratory distress or recurrent airway infections. One patient complained of abdominal pain. In one case, diagnosis was an incidental finding. No patient showed clinical features of a genetic syndrome.

\section{Mediastinal Teratoma}

There were 10 patients with teratoma, six males and four females. Median age at diagnosis was 3 years and 5 months (range: 0-16 years). Symptoms at onset are reported in Table I. In seven patients with mature teratoma and in three patients with immature teratoma (grade 2 in two patients according to GonzalesCrussi grading and grade 1 in one patient), $\alpha \mathrm{FP}$ and $\beta \mathrm{HCG}$ were normal at diagnosis (Table I). After preoperative imaging (CT or MRI), all patients were eligible for primary surgical resection, which was performed through posterolateral thoracotomic incision; major surgical issues that occurred were related to adhesion of lesions to the pericardium $(n=2)$ and lung $(n=2)$. In one patient, excision of both mass and pericardium was required. All patients are alive and under follow-up for periods ranging from 35 to 116 months (median: 91 months), without any evidence of either local recurrence or sequelae related to surgical treatment. Short-term sequelae recorded include pleural and pericardial effusions $(\mathrm{n}=1)$ and isolated pericardial effusion $(\mathrm{n}=1)$. All patients remain in complete remission after surgical resection only. No late sequelae have been recorded. Both overall survival (OS) and event free survival (EFS) are 100\% for these patients with a median follow-up of 89 months (range: 35-123).

\section{Malignant Mediastinal GCTs}

Ten patients (seven males and three females) had malignant mediastinal GCTs. Median age at diagnosis was 7 years and 9 months (range: 1 year to 17 years 8 months). Clinical characteristics are presented in Table II. None of these patients was eligible for primary resection of the tumor according to preoperative imaging. Biopsy of mediastinal lesions was then performed as primary procedure in nine patients (one CT-guided needle biopsy and eight minimally invasive surgical biopsies), while in one patient with life-threatening respiratory distress caused by the lesion and elevated serum levels of $\alpha \mathrm{FP}$ and $\beta \mathrm{HCG}(8,400$ and $360 \mathrm{ng} / \mathrm{ml}$, respectively), chemotherapy was started without prior histological examination.

In seven of 10 patients, biopsy specimens obtained were predominantly consistent for YST. $\alpha$ FP was elevated in all these patients at diagnosis (range: $21,000-84,000 \mathrm{ng} / \mathrm{ml}$ ), and also in the patient diagnosed with pleomorphic poorly differentiated GCTs $(50,000 \mathrm{ng} / \mathrm{ml})$. In one patient, choriocarcinoma was diagnosed on biopsy specimens. He presented with bilateral pulmonary metastasis and elevated levels of both $\alpha \mathrm{FP}$ and $\beta \mathrm{HCG}$ (610 and 460,000 ng/ml, respectively) at diagnosis, while the only patient with specimens predominantly consistent for seminoma had normal values of both tumor markers.

Seven of 10 patients had stage III disease, whereas three patients had stage IV disease with lung metastases in all of them and liver metastases in one patient.

Neoadjuvant cisplatin, etoposide, bleomycin (PEB) chemotherapy consisted of a combination of cisplatin, etoposide, and bleomycin (cisplatin $25 \mathrm{mg} / \mathrm{m}^{2}$ days $1-4$, etoposide $100 \mathrm{mg} / \mathrm{m}^{2}$ days $1-4$, bleomycin $15 \mathrm{IU} / \mathrm{m}^{2}$ day 2 ): eight of $10 \mathrm{pa}-$ tients received a median of four courses of PEB before delayed surgery (range: 2-6). In the two patients with symptomatic respiratory distress requiring mechanical ventilation, bleomycin was omitted to avoid the risk of adjunctive pulmonary toxicity; one patient had symptoms at diagnosis, and the other developed respiratory failure after diagnostic biopsy.

At the end of neoadjuvant treatment, two of three patients with stage IV disease achieved complete remission of metastases, whereas nine of 10 patients had normalized serum levels of $\alpha \mathrm{FP}$ and $\beta \mathrm{HCG}$.

Surgery was performed through posterolateral thoracotomic incision in five patients, while in five patients a median sternotomy was required. One patient underwent thoracotomy through a cervicosternal "clamshell" incision and surgical data for three patients are missing. In one patient, massive hemorrhage secondary to aortic leakage during dissection was reported, requiring repair under extracorporeal circulation.

In one patient, prolonged postoperative pneumothorax requiring Pleur-Evac ${ }^{\circledR}$ continuous chest drainage until the 15 th postoperative day was reported.

Nine of 10 patients are alive in complete remission. One patient with metastatic choriocarcinoma died of the disease at 25 months from diagnosis. Since he was refractory to the first-line treatment (six courses of PEB), he received the secondline treatment (two courses of ifosfamide, carboplatin, and 
TABLE II. Population of Malignant GCTs

\begin{tabular}{|c|c|c|c|c|c|c|c|c|c|c|}
\hline Patient & Sex & $\begin{array}{l}\text { Age at } \\
\text { diagnosis } \\
\text { (years, } \\
\text { months) }\end{array}$ & Symptoms & $\begin{array}{c}\alpha \mathrm{FP} \\
\text { at onset } \\
(\mathrm{ng} / \mathrm{ml})\end{array}$ & $\begin{array}{c}\beta \mathrm{HCG} \\
\text { at onset } \\
(\mathrm{mIU} / \mathrm{ml})\end{array}$ & $\begin{array}{c}\text { Biopsy } \\
\text { specimens } \\
\text { (predominant } \\
\text { malignant } \\
\text { component }^{\mathrm{a}} \text { ) }\end{array}$ & Stage & $\begin{array}{c}\text { Site of } \\
\text { metastases }\end{array}$ & Treatment & Outcome \\
\hline 1 & M & 13,0 & Abdominal pain & 610 & 460,000 & Choriocarcinoma & IV & Lung & $\begin{array}{r}\text { CT, Surg, } \\
\text { HDCT }\end{array}$ & DOD \\
\hline 2 & M & 17,0 & Chest pain & 21,091 & Normal & YST & III & No & CT, Surg & $\mathrm{RC}$ \\
\hline 3 & M & 3,5 & $\begin{array}{l}\text { Recurrent } \\
\text { pneumonia- } \\
\text { pleural effusion }\end{array}$ & 42,987 & Normal & YST & IV & Liver-lung & CT, Surg & $\mathrm{RC}$ \\
\hline 4 & $\mathrm{~F}$ & 1,0 & Fever & 22,449 & Normal & YST & III & No & CT, Surg & $\mathrm{RC}$ \\
\hline 5 & M & 14,0 & Worsening dyspnea & 8,400 & 360 & NA & III & No & CT, Surg & $\mathrm{RC}$ \\
\hline 6 & M & 17,0 & $\begin{array}{l}\text { Chest pain, } \\
\text { nonproductive } \\
\text { cough }\end{array}$ & 34,294 & Normal & YST & III & No & CT, Surg & $\mathrm{RC}$ \\
\hline 7 & M & 2,8 & Respiratory distress & 50,103 & Normal & $\begin{array}{l}\text { Epitelial-like } \\
\text { poorly } \\
\text { differentiated } \\
\text { tumor }\end{array}$ & III & No & CT, Surg & $\mathrm{RC}$ \\
\hline 8 & $\mathrm{~F}$ & 1,2 & Weight loss & 84,840 & Normal & YST & IV & Lung & CT, Surg & $\mathrm{RC}$ \\
\hline 9 & $\mathrm{~F}$ & 3,2 & Dyspnea & 50,000 & 28 & YST & III & No & CT, Surg & $\mathrm{RC}$ \\
\hline 10 & M & 12,3 & Respiratory distress & Normal & Normal & Seminoma & III & no & CT, Surg & $\mathrm{RC}$ \\
\hline
\end{tabular}

${ }^{a}$ Histology not representative of significant tumor sampling since obtained through fine-needle biopsy or minimally invasive surgical biopsy. YST, yolk sac tumor; CT, chemotherapy; HDCT, high-dose chemotherapy; Surg, surgery; DOD, dead of disease; RC, alive in complete remission.

etoposide), surgery on primary tumor, and high-dose chemotherapy with autologous stem cell support. Persistence of multiple residual pulmonary metastases, not eligible for gross total resection (adhesion to pulmonary hilum) thus precluding pneumonectomy, with normal serum levels of both markers, was also present. Relapse occurred 4 months after completion of therapy (new single pulmonary metastasis and increased blood $\beta \mathrm{HCG}$ ). He died from progression of disease, after surgery and third-line chemotherapy, at 12 months from relapse and 25 months from diagnosis.

With a median follow-up of 89 months (range: 35-123), the OS and EFS rates were both $90 \%$. In two patients, there was persistent residual postsurgical dysphonia as a sequela of mediastinal surgery. One of these patients also suffered a diaphragmatic eventration, which was surgically corrected 1 year after the end of treatment.

\section{DISCUSSION}

We report a series of children diagnosed with mediastinal teratoma and malignant GCTs. Patients with malignant GCTs were treated according to a single protocol during a 7-year period. The treatment for teratoma followed guidelines included in the AIEOP TCGM 2004. Patients were all treated in AIEOP centers using the same surgical approach. In our experience, the mediastinal site represents $5.4 \%$ of all GCTs registered from 2005 to 2013 in the AIEOP register; this percentage is in accord with data reported in the literature.[1,6]

GCTs represent approximately $3-4 \%$ of all cancers diagnosed in pediatric age. Only $2-4 \%$ of these tumors originate in the mediastinum. Malignant mediastinal GCTs are rarely encountered and case series have been reported by only a few centers. $[1-3,7]$

\section{Teratoma}

All 10 patients diagnosed with teratoma maintained a complete remission after total resection. Surgery represents the treatment of choice for mediastinal teratoma. Total surgical excision may result in a significant reduction in relapses and malignant evolution and should be performed in patients with immature components. In this respect, the percentage of immature elements within the tumor together with age of the patient and size of the mass are the most important risk factors for recurrence, and after surgical resection, close clinical monitoring with evaluation of tumor markers is mandatory for this population. [8,9] However, in the case of adherence of the lesion to great vessels, airways or nerves (phrenic, vagus) partial removal is the primary treatment to be considered in order to avoid potentially life-threatening or invalidating long-term sequelae.[10]

Regarding age distribution, Schneider and et al. reported an increased incidence of teratoma in patients at or soon after birth.[11] In fact, four of 10 patients in our series are younger than 12 months of age, confirming this age trend for this population.

\section{Malignant Tumors}

An OS of $>80 \%$ has been reported in different studies for patients under 18 years of age suffering from malignant mediastinal GCTs treated with a multidisciplinary approach based on surgery and chemotherapy.[8] Multidrug combinations vary in different long-term studies.[1-4,8] However, some studies on adult populations report worse outcome with survival rates at 5 years ranging from $23 \%$ to $47 \%$.[2,12-14] Reasons for these differences are still unclear. It may be speculated that the low number of studies in pediatric populations compared to adults 
may influence this finding. Nevertheless, some cytogenetic and molecular studies have shown profound differences in molecular biology between GCTs arising in infancy and those arising after onset of puberty despite identical histology, suggesting that pediatric GCTs stem from a different stage of germ cell development.[15-19]

A risk classification system for pediatric extracranial GCTs has recently been presented.[20] The authors found that age $>11$ years, ovarian stage IV disease, and extragonadal stages III and IV disease confer a significantly worse prognosis. The worst prognosis seems to affect males with age $>11$ years and extragonadal site (50\% 4-year EFS) while a borderline significance can be associated with histology other than YST. Even though 50\% of patients in our series are older than 11 years of age, only one had poor outcome. It could be speculated that our limited series confirms the trend of an increased prevalence of malignant mediastinal GCTs in males during adolescence (five of 10 patients were males older than 11 years of age). However, this trend may not be relevant given the small size of our population.

Regarding patients who underwent biopsy (nine of 10 with malignant GCTs), histology was consistent for only one tumor histotype even in the presence of elevated serum levels of both $\alpha \mathrm{FP}$ and $\beta \mathrm{HCG}$, indicative of a mixed neoplastic pattern. However, it must be borne in mind that our histology was based only on the results of minimally invasive procedures, and it is therefore possible that several patients had a mixed histology. Biopsy sampling could not be representative of all cellular populations expressed by mixed malignant GCTs due to the limited size of specimens, especially when obtained by fine-needle biopsy under ultrasound (US)/CT-guided procedures.[21] Furthermore, specimens resected after neoadjuvant chemotherapy may show either massive necrosis or residual teratoma due to a good clinical response to the treatment, thus precluding detection of all malignant components (embryonal carcinoma, choriocarcinoma, YST, and seminoma), which may have been present.[22]

In our study, patients with malignant GCTs were treated homogeneously. All patients, after diagnostic biopsy and/or tumor marker evaluation, underwent PEB chemotherapy. Bleomycin was omitted in two patients with severe respiratory distress. First-line therapy failed in only one patient with bilateral pulmonary metastases (patient number 1).

Nine of 10 patients with malignant tumor are in complete remission at a median of 97 months from diagnosis (range: 38 109).

Schneider et al. reported results of a cooperative study on 47 children with mediastinal GCTs, malignant in 26 cases, over a long period of 16 years. The combination of chemotherapeutic agents varied according to the different treatment protocols (MAligne KEImzelltumoren (MAKEI) 83/86, 89, and 96). All patients with teratoma were cured by tumor resection only. Patients with malignant GCTs had 5-year EFS and OS of $83 \%$ and $87 \%$, respectively.[7]

Billmire et al. reported results concerning 36 children with malignant mediastinal GCTs enrolled between 1990 and 1996 in the randomized intergroup pediatric oncology group (POG) trial to evaluate response rate and survival with chemotherapy using etoposide, bleomycin, and high or standard dose of cisplatin. The 4-year EFS and OS were $69 \%$ and $71 \%$, respectively. The authors suggested age $>15$ years as a prognostic factor for mortality due to tumor progression.[3]
Surgical removal is the treatment of choice for both teratomas and malignant GCTs of the mediastinum. [7,8] However, the surgeon may be faced with the challenging problem of adhesion of the lesion to major organs and vessels, which could lead to potentially life-threatening situations. Moreover, considering the risk of iatrogenic lesions of phrenic nerves, anesthesiological management is also of critical importance, since the loss of spontaneous respiratory activity during anesthesia may result in further compression of the airway by the mass.

Gross total resection represents the mainstay of treatment for malignant GCTs; [23] the choice of surgical procedure depends on the location of the tumor. Minimally invasive procedures, such as video-assisted thoracic surgery, may be indicated for small lesions with no evident adhesion to great vessels or surrounding structures. However, lobectomy and wedge resection are the procedures of choice for large tumors invading mediastinal organs. In such cases, to achieve an adequate resection, a combined procedure with extracorporeal circulation and cardiothoracic surgery may prevent catastrophic intraoperative events and improve the short- and long-term outcomes.[9,10,23]

In conclusion, we confirm the favorable outcome of children diagnosed with mediastinal malignant GCTs. Further studies would be necessary to highlight the subgroup of high-risk patients requiring treatment intensification from onset and to confirm age $>11$ years as a risk factor for worse prognosis as reported by others.[20] Moreover, a genetic analysis on tumor samples should be performed to distinguish subgroups of malignant GCTs that correlate with different clinical outcomes as suggested in some studies.[15,16]

\section{Acknowledgment}

The authors would like to thank Antonio Maria Zaccara, MD, Department of Nephrourology, Bambino Gesù Pediatric Hospital IRCCS, for his precious contribution to the revision of the manuscript.

\section{REFERENCES}

1. Yalçın B, Demir HA, Tanyel FC, Akçören Z, Varan A, Akyüz C, Kutluk T, Büyükpamukçu M. Mediastinal germ cell tumor in children. Pediatr Hematol Oncol 2012;29:633-642.

2. Liu Y, Wang Z, Peng ZM, Yu Y. Management of the primary malignant mediastinal germ cell tumors: Experience with 54 patients. Diagn Pathol 2011;9:33.

3. Billmire D, Vinocur C, Rescorla F, Colombani P, Cushing B, Hawkins E, London WB, Giller R, Lauer S. Malignant mediastinal germ cell tumors: An intergroup study. J Pediatr Surg 2001;36:1824

4. De Backer A, Madern GC, Pieters R, Haentjens P, Hakvoort-Cammel FG, Oosterhuis JW, Hazebroek FW. Influence of tumor site and histology on long-term survival in 193 children with extracranial germ cell tumors. Eur J Pediatr Surg 2009:18:1-6.

5. Lo Curto M, D'Angelo P, Cecchetto G, Klersy C, Dall'Igna P, Federico A, Siracusa F, Alaggio R, Bernini G, Conte M, De Laurentis T, Di Cataldo A, Inserra A, Santoro N, Tamaro P, Indolfi P. Mature and immature teratomas: Results of the first paediatric Italian study. Pediatr Surg Int 2007:23:315-322.

6. Eble JN, Sauter G, Epstein JI, Sesterhenn I. WHO classification of tumours. Pathology and genetics of tumours of the urinary system and male genital organs. Lyon, France: IARC Press; 2004.

7. Schneider DT, Calaminus G, Reinhard H, Gutjahr P, Kremens B, Harms D, Göbel U. Primary mediastinal germ cell tumors in children and adolescents: Results of the German cooperative protocols MAKEI 83/86, 89, and 96. J Clin Oncol 2000;18:832-839.

8. Takeda S, Miyoshi S, Ohta M, Minami M, Masaoka A, Matsuda H. Primary germ cell tumors in the mediastinum: A 50-year experience at a single Japanese institution. Cancer 2003;97:367-376.

9. Yalçın B, Demir HA, Tanyel FC, Akçören Z, Varan A, Akyüz C, Kutluk T,, Büyükpamukçu M. Mediastinal germ cell tumors in childhood. Pediatr Hematol Oncol 2012;29:633-642.

10. Chang CC, Chang YL, Lee JM, Chen JS, Hsu HH, Huang PM, Lee YC. 18 years surgical experience with mediastinal mature teratoma. J Formos Med Assoc 2010;109:287-292.

11. Schneider DT, Calaminus G, Koch S, Teske C, Schmidt P, Haas RJ, Harms D, Göbel U. Epidemiologic analysis of 1422 children and adolescents registered in the German germ cell tumor protocols. Pediatr Blood Cancer 2004;42:169-175.

12. Goss PE, Schwertfeger L, Blackstein ME, Iscoe NA, Ginsberg RJ, Simpson WJ, Jones DP, Shepherd FA. Extragonadal germ cell tumors: A 14-year Toronto experience. Cancer 1994;73:1971-1979.

13. Hidalgo M, Paz-Ares L, Rivera F, Lianes P, Huidobro G, Ruiz A, López-Brea M, Sanz-Ortiz J, López López J, Cortés-Funes H, Tabernero JM. Mediastinal non seminomatous germ cell tumors treated with cisplatin-based combination chemotherapy. Ann Oncol 1997;8:555-559. 
14. Fizazi K, Culine S, Droz JP, Kramar A, Théodore C, Ruffié P, Le Chevalier T. Primary mediastinal nonseminomatous germ cell tumor: Results of modern therapy including cisplatin-based chemotherapy. J Clin Oncol 1998;16:725-732.

15. Schneider DT, Schuster AE, Fritsch MK, Calaminus G, Göbel U, Harms D, Lauer S, Olson T, Perlman EJ. Genetic analysis of mediastinal nonseminomatous germ cell tumors in children and adolescents. Genes Chromosomes Cancer 2002;34:115-125.

16. Schneider DT, Schuster AE, Fritsch MK, Hu J, Olson T, Lauer S, Göbel U, Perlman EJ. Multipoint imprinting analysis indicates a common precursor cell for gonadal and nongonadal pediatric germ cell tumors. Cancer Res 2001;61:7268-7276.

17. Rajpert-De Meyts E, Nielsen JE, Skakkebaek NE, Almstrup K. Diagnostic markers for germ cell neoplasms: From placental-like alkaline phosphatase to micro-RNAs. Folia Histochem Cytobiol 2015;53:177-188.

18. Poynter JN, Bestrashniy JR, Silverstein KA, Hooten AJ, Lees C, Ross JA, Tolar J. Cross platform analysis of methylation, miRNA and stem cell gene expression data in germ cell tumors highlights characteristic differences by tumor histology. BMC Cancer 2015;15:769.
19. Looijenga LH, Van Agthoven T, Biermann K. Development of malignant germ cells: The genvironmental hypothesis. Int J Dev Biol 2013;57:241-253.

20. Frazier AL, Hale JP, Rodriguez-Galindo C, Dang H, Olson T, Murray MJ, Amatruda JF, Thornton C, Arul GS, Billmire D, Shaikh F, Pashankar F, Stoneham S, Krailo M, Nicholson JC. Revised risk classification for pediatric extracranial germ cell tumors based on 25 years of clinical trial data from the United Kingdom and United States. J Clin Oncol 2015;33:195203.

21. Akhtar M, al Dayel F. Is it feasible to diagnose germ-cell tumors by fine-needle aspiration biopsy? Diagn Cytopathol 1997;16;72-77.

22. Albers P, Weissbach L, Krege S, Kliesch S, Hartmann M, Heidenreich A, Walz P, Kuczyk M, Fimmers R; German Testicular Cancer Study Group. Prediction of necrosis after chemotherapy of advanced germ cell tumors: Results of a prospective multicenter trial of the German Testicular Cancer vanced germ cell tumors: Results of a prospect
Study Group. J Urol 2004;171:1835-1838.

23. Joly C, Deblock M, Desandes E, Geoffrois L. [Primary mediastinal germ cell tumors: A twenty years experience in a comprehensive cancer center]. Bull Cancer 2014;101:1067-1073. 